\title{
Kleines ABC der Pflege Begriffsbestimmung
}

Im kleinen ABC der Pflege stellen wir Ihnen wichtige Begriffe von E wie Expertenstandards bis T wie Tagespflege vor. Die meisten haben Sie sicher schon vielfach gehört, vielleicht wissen Sie aber nicht bei jedem hundertprozentig, was sich dahinter verbirgt.

Immer häufiger werden wir bei der Betreuung unserer meist älteren und multimorbiden Patienten mit Begriffen konfrontiert, von denen wir oftmals nicht genau wissen, was sich dahinter verbirgt. Wir haben deshalb für Sie ein kleines, nicht vollständiges, ABC der Pflege zusammengestellt, in welchem wir Ihnen einige dieser Begriffe erklären.

\section{Expertenstandards}

Mit verbindlichen Expertenstandards in der Pflege wird die Qualität der Einrichtungen und Dienste vergleichbar und verbessert. Die Standards sollen schrittweise entwickelt und von der Selbstverwaltung bis zum 30. September 2008 beschlossen werden. Damit wird Expertenwissen für den pflegerischen Einsatz konkret definiert und den professionell Pflegenden als Expertise im Alltag an die Hand gegeben. Expertenstandards sollen in Form eines Leitfadens aufbereitet werden.

\section{Kurzzeitpflege}

Die Kurzzeitpflege ist auf vier Wochen begrenzt. Sie kann etwa nach der Entlassung aus dem Krankenhaus in Anspruch genommen werden, wenn der Patient noch nicht in der Lage ist, selbstständig den Alltag zu meistern. Die Kurzzeit- pflege kann aber auch zur Überbrückung dienen, wenn Angehörige wegen Krankheit oder Urlaub die Pflege für einen gewissen Zeitraum nicht gewährleisten können. Die Leistungen für die Kurzzeitpflege werden im Zuge der Pflegereform schrittweise angehoben.

\section{Pflegestufe 0}

Bisher fielen vor allem Demenzkranke und geistig behinderte Menschen häufig durch das Raster der Pflegeversicherung, das sich nach körperlicher Einschränkung richtet. Menschen mit sogenannter „eingeschränkter Alltagskompetenz“, wie etwa Alzheimerpatienten, geht es jedoch körperlich vergleichsweise gut. Dennoch bedürfen sie der Betreuung und Zuwendung. Sie erhalten mit der Pflegestufe 0 statt der bisherigen 460 Euro nun bis zu 2400 Euro jährlich. Kommen körperliche Einschränkungen hinzu, gibt es daneben die monatlichen Geld- und Sachleistungen bei Vorliegen der Pflegestufen I bis III.

\section{Pflegestützpunkte}

Wer plötzlich Betreuung und Pflege für einen Angehörigen organisieren muss, stand bisher vor dem Problem, keine zentrale Anlaufstelle für alle Fragen zu haben. Diese Anlaufstelle gibt es mit der Reform der Pflegeversicherung in Form von Pflegestützpunkten. Diese bieten künftig adäquate Ansprechpartner, sogenannte Pflegeberater, und unterstützen die Betroffenen bei der Organisation der Pflege - angefangen von der Vermittlung von Pflegediensten, Haushaltshilfen bis hin zu guten Pflegeheimen oder anderen entsprechenden Betreuungseinrichtungen.

\section{Pflegezeit}

Schwere Krankheiten treffen Angehörige meist unvorbereitet und erfordern viel Zeit, um sich auf die neue Situation einzustellen und neue Strukturen zu schaffen. Kurzfristig können Arbeitnehmer im Zuge der neuen Reform der Pflegeversicherung eine Freistellung von zehn Tagen beanspruchen, um für die akut aufgetretene Pflegesituation eine bedarfsgerechte Pflege zu organisieren und eine pflegerische Versorgung in dieser Zeit sicherzustellen. Ferner haben Arbeitnehmer bei der Pflege von Angehörigen gegenüber dem Arbeitgeber einen Anspruch auf unbezahlte, aber sozialversicherte Freistellung von bis zu sechs Monaten, sofern der Betrieb mehr als 15 Beschäftigte hat.

\section{Senioren-WG}

Die Senioren-WG bietet die Möglichkeit, im Alter ein selbstständiges Leben zu führen und dabei mit anderen Menschen zusammen zu sein. Sind die Bewohner pflegebedürftig, können sie künftig ihren Anspruch auf grundpflegerische Leistungen und hauswirtschaftliche Versorgung bündeln und sich daraus ergebende Effizienzgewinne für den „Einkauf“ von zusätzlichen Betreuungsleistungen - beispielsweise Vorlesen - nutzen.

\section{Tagespflege}

Die Tagespflege wird häufig von Angehörigen in Anspruch genommen, die tagsüber berufstätig sind. Die Pflegebedürftigen werden meist morgens abgeholt und nachmittags zurück nach Hause gebracht. Die Tagespflege findet in Pflegeheimen oder in einer Tagesstätte statt. Pflegebedürftige erhalten dort ihre Mahlzeiten, befinden sich in Gesellschaft und werden körperlich und geistig aktiviert. Der Gesamtanspruch aus den Leistungen der häuslichen Pflege und den Leistungen der teilstationären Pflege wird mit der Pflegereform auf das 1,5-Fache des bisherigen Betrags erhöht.

Christa Tast, Vorsitzende fnb 